\title{
Peer Conversations for e-Learning in the Grid
}

\author{
Marc Eisenstadt, Jiří Komzák \\ Knowledge Media Institute, \\ The Open University, \\ Milton Keynes \\ MK7 6AA, UK \\ \{m.eisenstadt, j.komzak\}@open.ac.uk
}

\author{
Stefano A. Cerri \\ LIRMM, \\ CNRS \& Université Montpellier II, \\ 161, Rue Ada 34392 Montpellier Cedex 5, \\ France \\ cerri@lirmm.fr
}

\begin{abstract}
We take the view of 'learning as a conversational process', and argue that this can be extended to include the notions of peer-group interactions among students, tutors, and even artificial agents. This in turn lends itself to an approach to distributed conversation which builds upon modern Instant Messaging tools, and extends such tools to include what we call enhanced presence: a way of monitoring the availability of (and interacting with) fellow students and tutors at a distance. We describe a scenario being developed in the recently-started European Learning Grid Infrastructure 'ELeGl' project, deploying our own BuddySpace tools, and describe the novel contributions that this approach can bring to e-learning.
\end{abstract}

Conversational framework, e-learning, enhanced presence, grid.

\section{INTRODUCTION: ELEGI}

The European ELeGI project (European Learning Grid Infrastructure, www.elegi.org), started in February 2004, aims to provide an integrated framework for e-learning that builds upon practical and theoretical lessons learned over the past ten years, and harnesses the new promise of Grid technologies [1]. Our role in the ELeGI project is to elaborate, expand, and deliver a model of conversational processes and enhanced presence that we believe is central to the future of such e-learning frameworks.

Three activities have been influential in our work:

- It is apparent that many learning situations involve creative dialogues and conversations, and indeed strong theories of learning are built upon this concept

- The communicative aspect of the Internet is precisely one element that has proved exceptionally popular, as evidenced by the rapid growth of Instant Messaging and Peer-to-peer software

- The ability of web services to find and deploy intelligent agent software offers exciting promise for future intelligent applications

In this paper we provide the beginnings of one small part of our framework, namely the part concerned with enhanced presence, motivate it by reference to earlier influential theories, and illustrate our future directions by means of an evolving usage scenario.

\section{A CONVERSATIONAL FRAMEWORK}

One of the most influential theories in European Higher Education of the past 20 years is the Conversational Framework of Laurillard [10], who argues that learning can be viewed as a series of teacher-learner conversations taking place at multiple levels of abstraction. As summarized in [11]:

At the most general level of description, the learning process is characterized as a 'conversation' between teacher and student (see Figure 1), operating on two levels, discursive and interactive, the two levels being linked by the twin processes of adaptation and reflection.

In fact, the framework is more powerful than the simple diagram shows, because multiple styles of interaction are possible, as well as iterations within any type of interaction. As Laurillard et al describe the framework:

Students' work on an interactive resource will take place at the interactive level, where students' adapt their actions in the light of their current conceptual understanding, and discuss and reflect on their practical work in order to develop their conceptual understanding at the discursive level. Similarly, the interactive resource, expressing the 'teacher's experiential environment' is an adaptive response by the teacher who chooses it in the light of discursive level conversations with students, and a sense of what they need to do in order to learn the topic. Again, the teacher's reflection on what the students do during the interaction will drive their further discussions at the discursive level. 
TEACHER

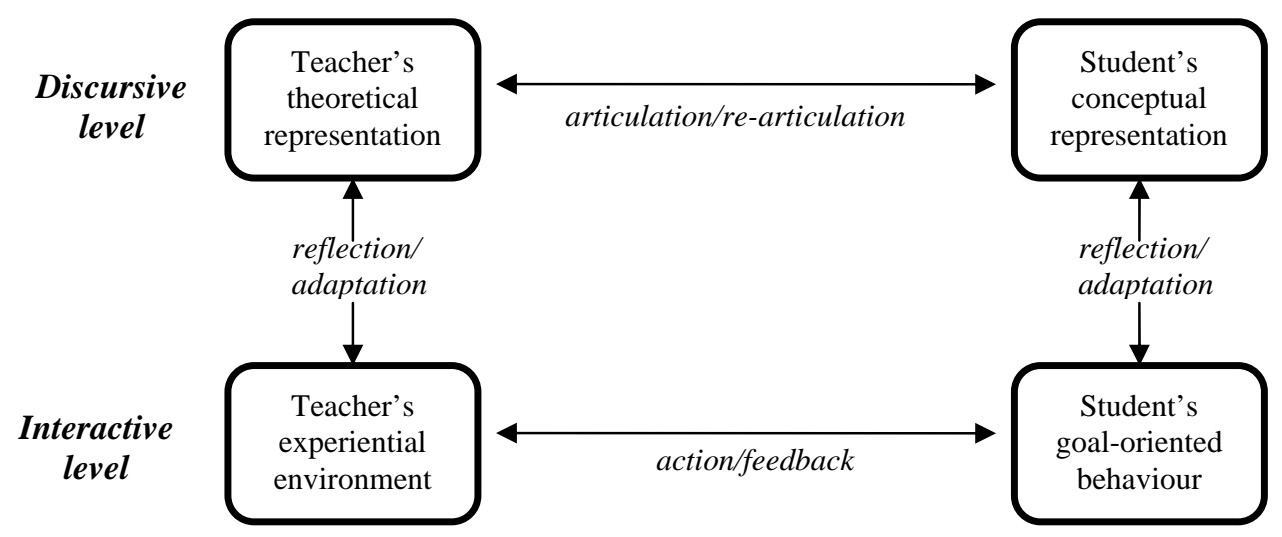

FIGURE 1: The conversational framework for the learning process (from [11])

As Britain and Liber argue [3], Laurillard's Conversational Framework serves as an excellent starting point for evaluating modern Virtual Learning Environments (VLE's). They argue that any VLE can be analyzed in terms of how well it supports discourse, whether it is adaptable, and how well it supports interaction and reflection - in other words the four key ingredients of the Conversational Framework (the extent to which an environment is discursive, adaptable, interactive, and reflective). In line with Laurillard's own description of the Conversational Framework, which emphasizes the possible iterations at each step, and multiple layers of abstraction possible, we have adapted Figure 1 by combining the analysis provided by Britain and Liber, and noted the scope for iterations, the involvement of agent software, and peer-to-peer interactions, all of which are presented in Figure 2.

TEACHER/AGENT

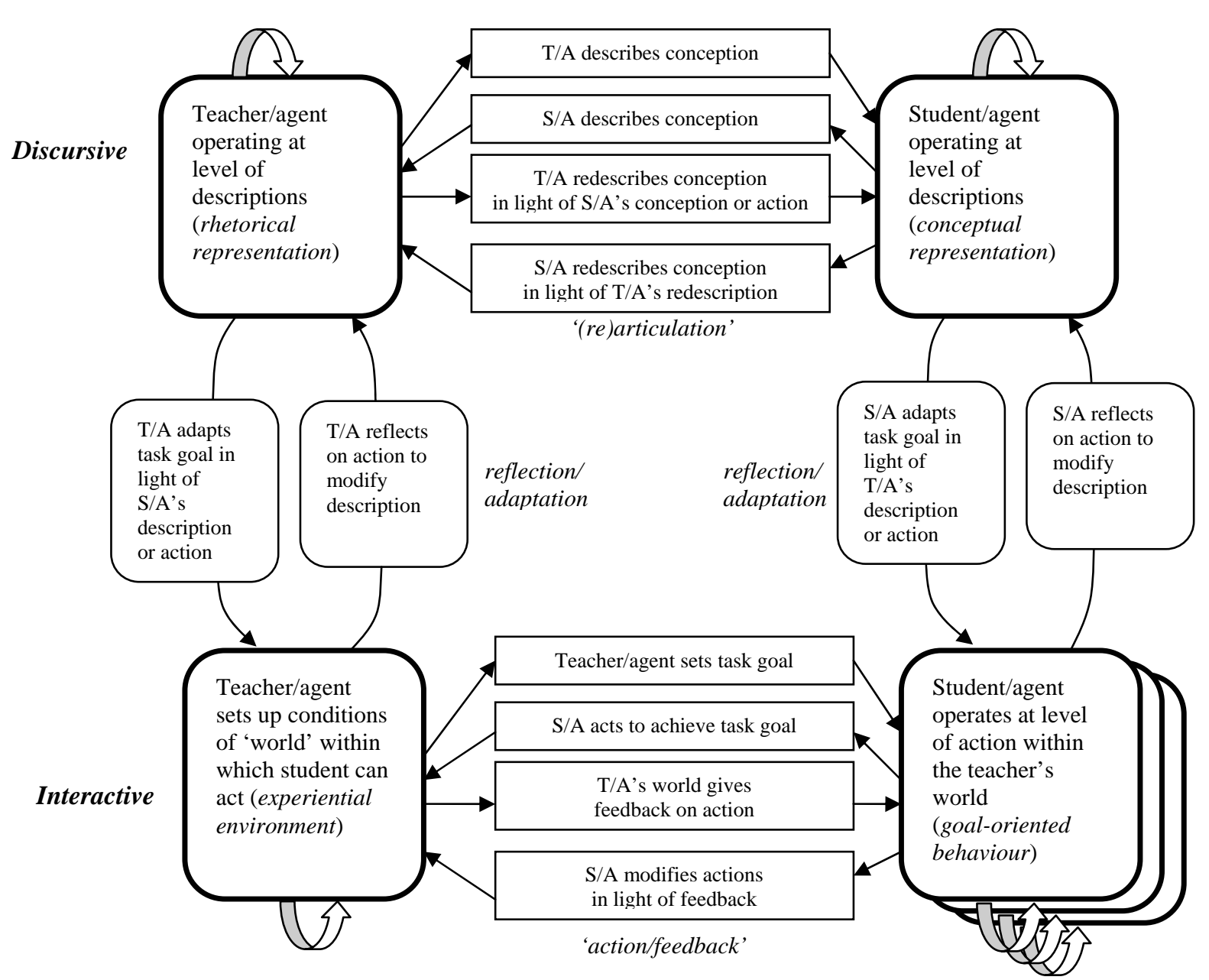

FIGURE 2: Extended conversational model, adapted from [3]

The original diagram of Britain and Liber shows the expansion of the four bi-directional arrows of figure 1 to illustrate the nature of the 'reflection' and 'adaptation' steps undertaken by the student and teacher (these are the up/down arrows), and also the articulation and action/feedback steps undertaken by the student and teacher (these are the left/right arrows). To this we have added (i) the 'wide curved arrows' in the four corners of the diagram, to highlight the iterative nature of every step (e.g. any action or thought process can involve multiple steps at different 
levels of abstraction); (ii) the 'Peer-to-peer' (P2P) multiple boxes in the lower right hand corner, to highlight the fact that peer group interactions (not only student-student but also teacher-teacher) are in fact fundamental to many learning scenarios, and (iii) the important caveat that any instance of Teacher T or Student S may also be viewed as an agent, and indeed may be either an artificial agent or a human agent, in an arbitrary mix (here labelled 'Teacher/agent $=\mathrm{T} / \mathrm{A}^{\prime}$ ' and 'Student/agent $=\mathrm{S} / \mathrm{A}$ '. The relationships among the possible T/A and S/A dyads is explored further in [5], whereas in the rest of this paper we concentrate on the peer group interactions.

How does the concept of 'Grid computing' fit into such a model? To answer this question, we draw a parallel between the essence of our approach and the initial reasons for conceiving and developing Grid technologies in the mid-1990's. The original observation was that facing a major increase of computational needs, for instance in the scientific community (for weather modelling and nuclear physics), there was a parallel increase of computational resources (machines and networks), for which many resources were proprietary and therefore not accessible directly nor on-demand. The original question was how to build a new infrastructure (the Grid) that would satisfy the computational needs in an on-demand fashion by exploiting the computational resources in a seamless way, not requiring 'physical possession' of the resource by the potential clients. That simple observation was based upon an optimization approach: previously idle machines would be used by those who would have needs, satisfying both (in fact, a win-win solution). Our 'conversational processes and enhanced presence' approach proposes, in exactly the same manner, to combine latent and explicit learning needs with potentially available teaching/learning resources able to satisfy those needs in a kind of 'human grid' - in this case offer and demand are combined, driven by the demand and enabled by the infrastructure. This is a first approximation to our proposed 'Open Grid Human Service Architecture' in the ELeGI project, where we study conversational processes among humans (mediated by machines) trying to offer human resources to learners in an on-demand (or 'as needed') manner.

\section{ENHANCED PRESENCE AND MESSAGING WITH BUDDYSPACE}

The notion of peer-group interactions, highlighted in the lower right corner of Figure 2, is critical to many learning interactions. Johnson and Johnson [9] and numerous others have described the benefits that accrue from activities such as joint problem solving and related peer interactions that take place in the presence of other students. But in an e-learning situation, what exactly does it mean to be 'in the presence of other students'? The notion of 'presence' must therefore be extended, because the other students, and indeed the teacher or tutor, may not be physically present: instead they are only virtually present. Our belief is that simply being able to see the other person, although obviously useful, is neither necessary nor sufficient to impart a sense of presence: rather it is more of a mental state that depends on knowing the other person is available, even at a distance, and this can be de-coupled from 'visibility'. This mental state is crucial for what we called 'enhanced presence' (see the Step 5 in the scenario).

As we described in an earlier TelEduc03 paper [8] and in [2], 'enhanced presence' builds upon the ideas of 'peripheral awareness of others' made so popular by Instant Messaging, and extends it to include a variety of 'desktop radar' or 'dashboard' capabilities to facilitate collaborative interactions. Our research in this area has led to the development of BuddySpace, an Instant Messaging environment (based on the Jabber/XMPP protocol) with both client and server functionality extended to enhance presence awareness. Specifically, it introduces automatic roster ('buddy list') construction and intelligent service discovery on the server and the graphical visualization of people and their presence states on an image, geographical or conceptual map (as in Figure 3 later in the paper). This allows for multiple views of collaborative workgroups: the immediacy gives users a snapshot of a virtual organization, which is critical in modern learning organizations: We know from [16] that presence awareness increases emotional well-being, and from [12] that users benefit from knowing who else is around via presence and messaging tools. In a virtual classroom setting, the instant message capabilities of BuddySpace naturally provide a "backchannel" to the classroom, for example, conveying URLs of documents discussed or as a non-disrupting communication. For distributed activities involving videoconferencing, the presence of individuals gives an extra indication of co-location (especially if the videoconferencing technology is failing). The back-channel can also be used for meeting control tasks, such as queuing of speakers and voting on issues.

In an e-learning context, BuddySpace leverages the overwhelming power of social cohesiveness that can be brought about by knowledge of the presence and location of others in both real and virtual spaces, in the style argued persuasively by [13]. We know also from our own informal observations that this kind of presence awareness is used by students to locate resources, for quick exchange of information and to organize meetings either online or face-to face. Indeed we argue that Enhanced Presence is much more than just 'messaging' and 'maps'. In particular, we aim to provide tools that enable us to express the entire situated context of the learner, which is clearly a lot more than just 'location X' and 'online' or 'offline'. The learner's current state of mind, including goals, plans and intentions, must be understood, as well as the way this connects with ongoing activities and devices accessible to the learner. As these are made explicit, plausible inferences can be drawn about what the leaner wants and needs to know, and this gives us an important 'foot in the door' for addressing the problem of delivering the right knowledge to the right people in the right place at the right time. So far, this notion of 'right knowledge' has been nothing more than a Knowledge Management 'slogan', but our belief is that Enhanced Presence capabilities can make this dream a reality. For instance, an important precondition for 'the right knowledge' consists in having access to someone (a human) who eventually would possess it and would be 
available for delivering it as a service. Enhanced presence aims at answering exactly this question: 'Who out there somewhere can help me now, or has solved a similar or related problem?' Note that in this model, humans are of course potential service providers too, and therefore any 'service discovery tools' must also be capable of discovering relevant people.

\section{A SCENARIO DEPLOYING ENHANCED PRESENCE FOR E-LEARNING}

Below we describe an e-learning scenario, based on actual Open University BuddySpace experiences, involving groups of students enrolled in 3rd-level Spanish language courses at a number of universities across the UK. They are asked to work in groups of 4 collaboratively to edit, design, and produce a Spanish-language web-based newsletter, containing items of interest to the Spanish-language community. Sub-tasks include deciding what the scope of the content should actually be (e.g. sports, news, culture) - this is a deliberately open-ended assignment. Students work together to produce the newsletter, but must ultimately produce their own write-up for their own tutor. Key players in the scenario include the students, their tutors, and network administrators at several universities. The scenario comprises the following steps, elaborated in detail further below:

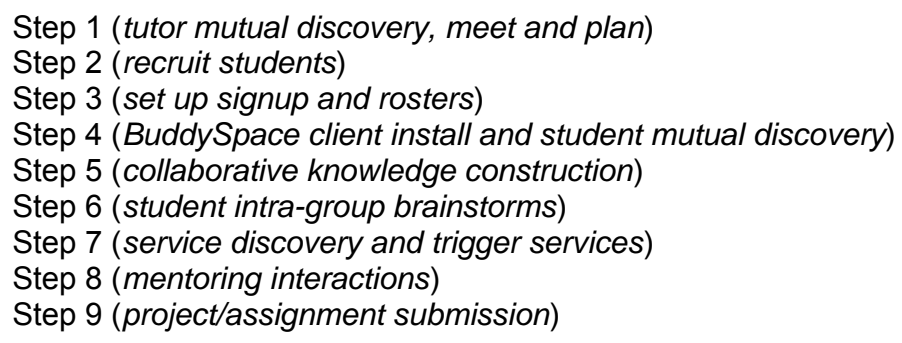

Below we expand the nine steps and illustrate the aspects of enhanced presence, conversation, and service discovery that are important for e-learning.

\section{THE EXPANDED SCENARIO}

\subsection{Step 1 (Tutor mutual discovery, meet and plan)}

The tutors are part of a UK-wide Spanish Language Universities Group. They 'meet' in a regularly-scheduled multi-user chat-room using BuddySpace, having first located each other through BuddySpace's 'semantic matchmaking' service, which identified them according to their user profiles as each (a) interested in CALL (Computer Assisted Language Learning), (b) experts in several relevant technologies, (c) fluent in or instructors in Spanish. A quick chat/brainstorm one evening in real-time results in a coherent project suggestion for their students.

\section{Important benefits for e-learning:}

- $\quad$ Automatically constructed profiles: Each institution/university can automatically fill in some of the fields in personal profile of each employee according to his/her job description and previous experience mentioned in her/his CV. More personal details can be entered by the tutors later.

- Matchmaking: Work on a common project requires participating tutors to have several skills, but also interests. The matchmaking itself can be done by finding similarities between requirements and tutors' profiles.

- Chat/brainstorm: During the short discussion the tutor can learn more about experience of others in teaching the particular part of the subject. They also negotiate the best formulation of the project: suggestions $->$ evaluation $->$ modifications...

\subsection{Step 2 (recruit students)}

The tutors inform their respective students of the proposed task, and ask students to register for a new BuddySpace group, to grant authorization for their thumbnail photos to be used, and for coarse-grained locations (and possibly other conceptual information) to be made visible to other group members.

Important benefits for e-learning:

- $\quad$ Opt-in: Students need to agree to take part. They also confirm what information can be used to populate their profiles.

- Zones-of-trust: Access to all information (e.g. in profiles) can be set to different levels of detail for groups or individuals. Thus, for each course/project group, the visible profile can be different.

\subsection{Step 3 (set up signup and rosters)}

The tutors ask the network admin people to set up custom rosters for these groups of BuddySpace users. The servers synchronize with each other, so that students see all remote group members.

\section{Important benefits for e-learning:}

- Automatic rosters: Automatic generation of rosters (contact lists) is one of the ways to push customized contextual information to the students. It's an easy way how to learn who the fellow students are. 


\subsection{Step 4 (BuddySpace client install and student mutual discovery)}

Students install/launch BuddySpace, inherit their automatically-generated roster group, and fill in a user profile in their client, specifying from among recognized interest areas that have been pulled form the server. They then seek out fellow students and self-form small groups/teams.

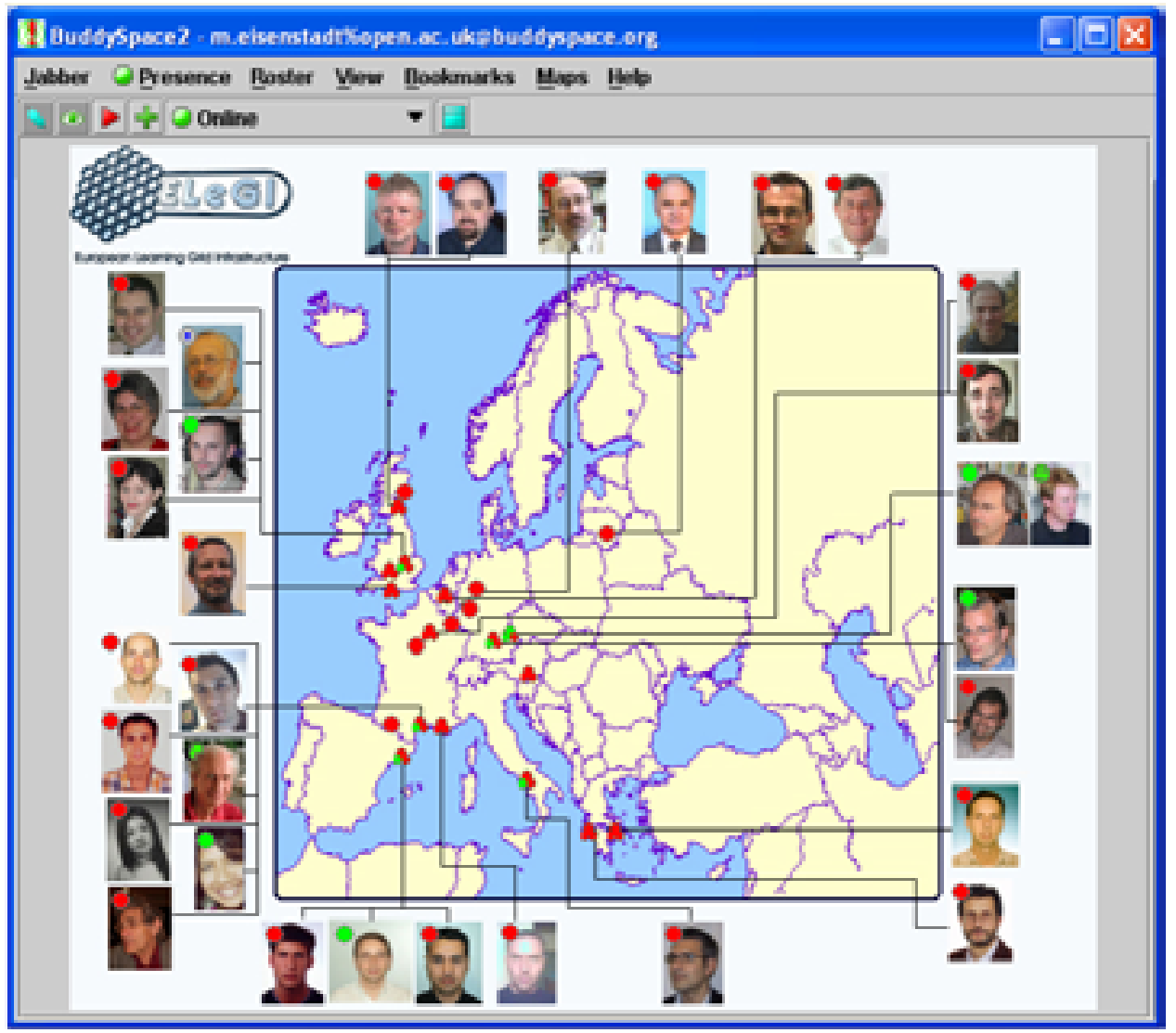

FIGURE 3: Automatically-generated map (in this case showing ELeGI users of BuddySpace), to illustrate how existing technology would be deployed in the Scenario. Green (darkest) dots depict currently-online ('available') users.

\section{Important benefits for e-learning:}

- Custom profiles: Entering a profile can be thought of not only as 'filling in a database', but more significantly as providing a 'benign agent' with a collection of significant facts about the person. Thus, it can not only be just filling-in a form, but also more generic learning with definitions of new terms. Using machine learning, an agent can deduce even more information from user's behaviour (e.g. on-line patterns) and conversations with others (e.g. topics).

- Matchmaking: Students can form groups in different ways - looking for fellows with similar interests, living in the same area, previous courses, and so on.

\subsection{Step 5 (collaborative knowledge construction)}

While studying the course materials, a student may be unclear about some principles or terms, or may want to discuss them with other students. The student can discuss this during a scheduled group session, or use the enhanced presence status to find other students who are studying the same materials or working on similar materials (e.g. using a trigger service described further in Step 7) and start an ad-hoc chat with them. The interaction with the others can take two forms: one of the students can take the role of the tutor and explain the principle (while learning to formulate ideas and express him/herself) while others comment and discuss whether that understanding is correct; alternatively, all students may come up with possible explanations and opinions, which help others to see different perspectives or understand the underlying processes and rules. In these cases, we believe that common or collaborative knowledge (understanding) is actually constructed during the chat.

\section{Important benefits for e-learning:}

- Enhanced presence: the entire context of the learner includes not only his/her state of mind (e.g. goals, plans and intentions), but also current and ongoing activities and devices accessible to the learner.

- Ad-hoc chat: Chat can be triggered by the current needs of the student or state-change of some of his/her fellow students. The enhanced presence status helps to find a matching counterpart for this type of chat (somebody who is suitable, willing and ready to chat).

\subsection{Step 6 (student intra-group brainstorms)}

The students have formed into 12 groups of 3 and one group of 4, with tutor permission. Each group now begins the real task, starting to brainstorm about the issues. Most groups do this within BuddySpace multi user chat rooms, though some use telephone conference calls and other existing VLE tools, including our own FlashMeeting 
and Hexagon; the advantage of using BuddySpace is that it provides a layer of scaffolding, monitoring, and intelligent agent intervention unavailable with other approaches.

Important benefits for e-learning:

- Chat/brainstorming: Again, students can learn more about others - who is more willing or 'suitable' to do each task. Then they negotiate the best way to proceed: suggestions -> evaluation -> modifications -> voting...

- Agent intervention: dialogue patterns can be detected and used for 'benign intervention' and assistance with service discovery.

\subsection{Step 7 (service discovery and trigger services)}

One group decides to create a blog style newsletter about (say) the forthcoming Spanish elections, using recommended VLE tools, but gets confused due to a lack of understanding of all the political acronyms such as 'PP'. Using BuddySpace they find a foreign language acronym-service, which expands acronyms immediately in a separate chat dialogue. Another valuable service they find is a 'trigger' service that will alert them, via instant (service) message directly to BuddySpace, of 'hot stories' pertinent to their election newsletter. They can bring these up in compact form via an RSS feed within BuddySpace, and launch full stories of interest with one click directly in a browser window.

\section{Important benefits for e-learning:}

- Services/resources discovery: Services and resources can be either GRID or Jabber-based, or both. Here we talk about the Jabber ones, although they can use similar description formats (from WSDL to IEEE LOM). The Jabber Publish/Subscribe protocol allows users to browse a tree hierarchy of published nodes and view their properties. The Jabber Service discovery protocol is similar.

- Use of services: Services generally can use their own protocol. It can be anything from natural language to filling of forms or even telephone-like "press 1 for...". The acronym service could provide a simple chat-like interface, answering queries such as "explain FAQ" and possibly allowing the entering of new acronyms.

- Trigger services: Trigger services allow users to define a set of conditions and actions to be performed when the conditions are fulfilled. The conditions can include presence of specific people, state of a device, newly published items (e.g. RSS feed or Pub/Sub) or other defined properties (e.g. location) in certain range (e.g. close to my location). Triggers can send a message, change their own properties or behaviour, and so on. In the language of agents, a trigger service is an agent observing its environment and acting in specific situations. Again the conditions can be fuzzy or even automatically learned.

\subsection{Step 8 (mentoring interactions)}

During every 4th session one of the tutors 'drops into' the ongoing BuddySpace session, to observe and comment on the way things are going. Tutors also send out their own IM alerts with updates, thoughts, and any stop-press info, as these are deemed to be just-in-time and ephemeral.

\section{Important benefits for e-learning:}

- Chat/teaching: Tutors observing the behaviour of students can learn much about skills and problems of students during the task solving. They can immediately provide explanations and feedback - specific mentoring actions in the style of the STROBE model proposed by LIRMM would be embedded in the detailed expansion of this step.

\subsection{Step 9 (project/assignment submission)}

The students complete their newsletters, submit their assignments via a custom form no their respective e-learning sites and flash a BuddySpace alert around to their cohorts about two celebration parties to take place: one virtual and one real, at the geographical centre of their respective locations.

\section{Important benefits for e-learning:}

- Publishing: The assignments can be published using Jabber's own mechanism which also allows access permission control, in line with the 'zones of trust' described earlier. It's generally a way to provide information (e.g. assignment, location or profile) to certain set of people (subscribers).

- Trigger services: See above.

- Social chat: Celebrations!!! Learning in a social context could be promoted under this heading.

Although the above steps only depict a 'scenario' for which we are currently developing the suitable services to be delivered throughout the recently-started ELeGI project, all of the steps in the scenario are based on capabilities with which we have extensive experience at The Open University, including pilot studies already undertaken with hundreds of students in the Department of Languages [14], and more general derived from our practical experiences delivering distance learning to 200,000 students annually [6]. We therefore believe it is a plausible route for delivering realistic next-generation services for our students.

\section{CONCLUSIONS}

'Conversation' is clearly an important ingredient of the learning process: indeed, even if you disagree with the underlying model put forward by Laurillard and her colleagues, it is still clear that conversations occur at many levels of abstraction during learning! We have argued that in light of the importance of conversation for learning, then modern communication media such as Instant Messaging are a natural and important focus for e-learning. But such media are not normally considered to be supportive of extended tutorial dialogues, and it is therefore 
important to emphasize that 'enhanced presence' is much more than just 'messaging': it is an environment for representing the mental states of all participants, and a vehicle for alerting peer groups at a distance in ways that facilitate many different styles of interaction.

We have described our motivation for investigating enhanced presence and messaging with tools such as BuddySpace, and presented a scenario which looks at a very coarse-grained view of an entire distance-learning project life-cycle. Indeed, this life-cycle (nine steps) represents a much more ambitious focus than typical shortterm e-learning dialogues. We have argued that there are numerous benefits for the learner, particular in the innovations that can be delivered through the deployment and discovery of services such as matchmaking and trigger alerts. Because our scenario is based on years of experience with hundreds of thousands of distance learning students at The Open University, we believe it is realistic, and look forward to pursuing our vision and delivering enhanced presence services within the ELeGI project.

\section{ACKNOWLEDGEMENTS}

This work is supported by the European Community under the Information Society Technologies (IST) programme of the 6th Framework Programme for RTD - project ELeGI, contract IST-002205. This document does not represent the opinion of the European Community, and the European Community is not responsible for any use that might be made of data appearing therein.

We are grateful to our colleagues Martin Dzbor (KMi, Open University) for significant input into the concepts, design, and implementations underlying BuddySpace, and Joost Breuker, Clement Jonquet, and Philippe Lemoisson (LIRMM, Université de Montpellier) for significant input into the concepts, designs, and implementations underlying our work on conversation.

\section{REFERENCES:}

[1] Allison, C., Cerri, S.A., Gaeta, M., Ritrovato, P. and Salerno, S. (2003) Human Learning as a Global Challenge: European Learning Grid Infrastructure. In Varis, T., Utsumi, T. and Klemm, W. (eds.), Global Peace Through the Global University System, RCVE, Tampere, 465-488

(http://www.terena.nl/conferences/tnc2004/core getfile.php?file id=576)

[2] Bachler, M, Buckingham Shum, S., Chen-Burger, J. Dalton, J., De Roure, D., Eisenstadt, M., Komzak, J., Michaelides, D., Page, K., Potter, S., Shadbolt, N., and Tate, A. (2004) Collaboration in the Semantic Grid: a Basis for e-Learning. In 7th Int. Conf. On Intelligent Tutoring Systems (ITS 2004) - Workshop on GRID Learning Services (GLS04) (http://www.info.uqam.ca/\%7Enkambou/gls/gls04.htm)

[3] Britain, S. and Liber, O. (2000) A framework for the pedagogical evaluation of virtual learning environments, JTAP Report 041, University of Wales - Bangor, UK (http://www.jisc.ac.uk/jtap/htm/jtap 041.html)

[4] Buckingham Shum, S., De Roure, D., Eisenstadt, M., Shadbolt, N., and Tate, A. (2002) CoAKTinG: Collaborative Advanced Knowledge Technologies in the Grid. Proceedings of Second Workshop on Advanced Collaborative Environments, 11th IEEE Int. Symposium on High Performance Distributed Computing, Edinburgh, 24-26 July.

[5] Cerri, S.A., Eisenstadt, M. and Jonquet, C. (2003) Dynamic Learning Agents and Enhanced Presence on the Grid. Proceedings of the 3rd International Workshop on GRID Infrastructure to Support Future Technology Enhanced Learning (IST-2001-38763), Berlin, December. British Computer Society Electronic Workshops in Computing (BCS eWiC); Category: Grid computing (http://ewic.bcs.org/conferences/2003/3rdlege/index.htm)

[6] Daniel, J.S. (1997) Mega-Universities and Knowledge Media: Technology Strategies for Higher Education. Kogan Page, London.

[7] Eisenstadt, M., and Dzbor, M. (2002) BuddySpace: Enhanced Presence Management for Collaborative Learning, Working, Gaming and Beyond. Proceedings of JabberConf Europe 2002, 11-13 June, Munich Germany.

[8] Eisenstadt, M., Komzak, J., and Dzbor, M. (2003) Instant messaging + maps = powerful tools for distance learning. Proceedings of TelEduc03, May.

[9] Johnson, R. T., \& Johnson, D. W. (1986) Action research: Cooperative learning in the science classroom. Science and Children, 24, 31-32.

[10] Laurillard, D. (2002) Rethinking University Teaching. Routledge, London (2nd edition, 2002).

[11] Laurillard, D., Stratfold, M., Luckin, R., Plowman, L., and Taylor, J. (2000) Affordances for Learning in a NonLinear Narrative Medium. Journal of Interactive Media in Education, 2000 (2).

(http://www-jime.open.ac.uk/00/2/laurillard-00-2-paper.html) 
[12] Nardi, B.A., Whittaker, S., Isaacs, E., Creech, M., Johnson, J., and Hainsworth, J. (2002) Integrating communication and information through ContactMap. Communications of the ACM, 45:4, 89-95.

[13] Rheingold, H. (2002) Smart Mobs: The Next Social Revolution. Cambridge, Mass., USA: Perseus.

[14] Rossade, K-D. (2003) Audio-graphic-conferencing and Instant Messaging in language learning. Proceedings of the 8th biennial conference of the International Association for Language Learning Technology, Ann Arbor, Michigan, USA, 17-21 June, 2003.

[15] Scott, P., and Eisenstadt, M. (1998) Exploring telepresence on the Internet: the KMi Stadium Webcast experience. In Eisenstadt, M. and Vincent, T. (eds.), The Knowledge Web: Learning and Collaborating On The Net, Kogan Page, London.

[16] Whitelock, D., Romano, D.M., Jelfs, A., and Brna, P. (2000) Perfect Presence: What does this mean for the design of virtual learning environments? Education and Information Technologies, 5:4, 277-289. 